\title{
Dosis de radiación en pacientes sometidos a angiotomografía coronaria y score de calcio
}

\section{Radiation dose in patients undergoing coronary CT angiography and calcium score}

Autores:

Palacio Adriana1; Maldonado Samanta1; Mora Paula1; Lozano Alexander² Rodríguez Felipe $^{3}$

${ }^{1}$ Area Biomédica, Universidad Técnica Particular de Loja, Loja-Ecuador.

${ }^{2}$ División de Tomografía Computada, Hospital Clínica San Agustín, Universidad Técnica Particular de Loja, Loja-Ecuador.

${ }^{3}$ Departamento de Imagen Hospital Clínica San Agustín, Loja-Ecuador.

\begin{abstract}
Resumen: El uso de angiotomografía de arterias coronarias y el score de calcio ha incrementado notablemente debido a su gran capacidad para la evaluación de pacientes con enfermedad arterial coronaria. En el presente estudio observacional se determinan las dosis de radiación efectiva que recibieron los pacientes a través de cada uno de estos estudios. Se analizaron los estudios de angiotomografía de coronarias y score de calcio realizados en la división de Tomografía Computada del Hospital Clínica San Agustín en el período comprendido entre diciembre del 2017 y septiembre del 2020. Se calculó la dosis efectiva, utilizando el producto longitud dosis por el factor de conversión de Monte Carlo de 0,014 mSv. mGy* $\mathrm{cm}$. La dosis de radiación efectiva total de los pacientes que se realizaron únicamente score de calcio fue de $1,01 \mathrm{mSv} \pm 0,12 D S$, aquellos que se realizaron angiotomografía de coronarias fue de $11,71 \mathrm{mSv} \pm 1,77 D S$ y de $12,04 \mathrm{mSv} \pm 1,65 \mathrm{DS}$ para quienes se realizaron ambos estudios. Aunque la dosis efectiva de angiotomografía de coronarias y la de score de calcio fue similar a la de otros estudios, manteniéndose dentro de los parámetros recomendados por organismo reguladores, es razonable en la medida de lo posible realizar ajustes de las variables para disminuir la radiación ionizante que reciben los pacientes.
\end{abstract}

Abstract: The use of coronary artery angiography and calcium score has increased due to its great capacity for evaluating patients with coronary artery disease. In this observational study, the effective radiation doses received by the patients are determined through a calcium score study and coronary angiography, through the calculation of effective radiation. The coronary angiography and calcium score studies performed in the Computed Tomography division of the Hospital Clínica San Agustín in the period between December 2017 and September 2020 were analyzed. The effective dose was calculated, using the dose length product by the Monte Carlo conversion factor of $0.014 \mathrm{mSv}$. $\mathrm{mGy}{ }^{*} \mathrm{~cm}$. The total effective radiation dose of the patients who underwent calcium score alone was $1.01 \mathrm{mSv} \pm 0.12 S D$, those who underwent coronary CT angiography was $11.71 \mathrm{mSv} \pm 1.77 \mathrm{SD}$ and $12.04 \mathrm{mSv} \pm 1,65 S D$ for whom both studies were conducted. Although the effective dose of coronary CT angiography and calcium score was like that of other studies, and was within the parameters recommended by regulatory bodies, it is reasonable as far as possible to adjust the variables to reduce radiation ionizing that patients receive.

\section{Introducción}

A partir de su debut en la década de 1970, el uso de la tomografía computarizada (TC) ha incrementado rápidamente, estimándose que desde el año 1996, se cuenta con aproximadamente 26 y 64 tomógrafos por cada millón de habitantes en países como Estados Unidos y Japón, respectivamente; de forma que en el primero, hasta el año 2007 se han registrado más de 62 millones de tomografías computarizadas realizadas anualmente. Este notable incremento se ha debido en gran parte a los avances tecnológicos con enfoque conservador, que se han visto reflejados en la creación de estudios no invasivos para el cribado de pacientes adultos asintomáticos con factores de riesgo que conllevan a patologías que implican una elevada tasa de morbilidad y mortalidad ${ }^{1}$.

La TC tiene relativamente dosis de radiación más altas al compararse con los estudios de imagen convencionales que emiten radiaciones ionizantes, razón por la cual, su uso frecuente se ha asociado con un
Palabras clave: Dosis de radiación, angiografía por tomografía computada, Método Monte Carlo, seguridad del paciente.

Key words: Radiation dose, computed tomography angiography, Monte Carlo method, patient safety.

Comité de ética: Este estudio no requiere comité de ética, se trata de una revisión retrospectiva.

Correo para correspondencia del autor principal:

aslozano@utpl.edu.ec

Fecha de recepción:

10 de febrero de 2021.

Fecha de aceptación:

05 de junio de 2021. elevado riesgo de cáncer para los pacientes. En Estados Unidos, una persona promedio se expone a un $18 \%$ de radiación artificial, de la cual un $56 \%$ corresponde a radiación dada por estudios de imagen diagnósticos, entre los cuales destacan los estudios de imagenología cardíaca e intervencionistas, responsables de aproximadamente el $40 \%$ de la dosis efectiva acumulada².

Diversos estudios estiman que el umbral aceptado sobre el cual aumenta el riesgo de malignidad son $100 \mathrm{mSv}$, entre los estudios tomográficos que emiten una considerable dosis de radiación se encuentran las tomografías de abdomen que con un estudio simple y contrastado alcanzan alrededor de $15,4 \mathrm{mSv}^{3}$ y la angiotomografía de arterias coronarias (ATCC) que, por su parte, puede ir de 12 a $30 \mathrm{mSv}$.

Es necesario recordar que la dosis de radiación es acumulativa, es decir, que toda la dosis de radiación recibida en dicho lapso aumenta el riesgo de efectos no deseados ${ }^{4}$. 
Aunque los riesgos individuales no son significativos, al analizar la población irradiada de forma global, pueden llegar a ser un problema de salud pública en el futuro, debido al número creciente de pacientes con múltiples enfermedades crónicas que son expuestos a una cantidad variable de exámenes de imagen.

La falta de un historial que determine a cuanta radiación se ha sometido un paciente aumenta la probabilidad de que los efectos determinísticos que afecten la salud de los pacientes sean mayores.

En nuestro país no existen guías que propongan criterios específicos para la indicación de métodos de imagen, ni parámetros que modulen la dosis de radiación empleada, adicionalmente, dentro de los protocolos aplicados, no se incluyen los reportes dosimétricos de la radiación que ha recibido un paciente.

El objetivo de llevar un control dosimétrico está orientado a prevenir y establecer un límite para la aparición de efectos nocivos, por lo tanto, tenemos una población expuesta a cantidades importantes pero indocumentadas de radiación ionizante que probablemente serán responsables de iatrogenias a largo plazo ${ }^{5}$.

\section{Materiales y Métodos}

Previa aprobación institucional se accedió a los estudios de tomografía cardíaca. Se realizó un análisis retrospectivo transversal de pacientes que se realizaron una angiotomografía coronaria (ATCC) y/o score de calcio (SC) en el periodo de diciembre del 2017 a septiembre del 2020 en el Hospital Clínica San Agustín en Loja (HCSA), Ecuador.

Se incluyeron a los pacientes con edad mayor o igual a 18 años que contaban con el estudio dentro del sistema de almacenamiento y transferencia de imágenes (PACS) con el respectivo informe de dosis.

Los criterios de exclusión se aplicaron para los estudios de imagen que no contenían la identificación del paciente o el informe de dosis y para aquellos que fueron realizados en menores de 18 años.

Los pacientes se clasificaron en tres categorías dependiendo de tipo de estudio que se realizaron: aquellos que contaban únicamente con un SC, quienes tenían una ATCC y los pacientes que se realizaron ambos estudios simultáneamente.

Para todos los estudios realizados se utilizó un tomógrafo marca PHILIPS Brillance 64 canales activo desde el año 2017. El equipo de Tomografía del HCSA recibe mantenimiento preventivo de forma regular 2 veces al año por parte de los proveedores y el oficial de seguridad radiológica (OSR) realiza una valoración técnica anual para garantizar el cumplimiento y la garantía de calidad.

Las exploraciones se realizaron con el paciente en posición decúbito supino en apnea inspiratoria, en fase simple y empleando contraste intravenoso, con técnica helicoidal.

El rango de exploración fue en sentido cráneo caudal, los parámetros de adquisición de kV y mAs se ajustaron a la talla y peso de los pacientes, los pacientes con frecuencias cardíacas estables permitieron el uso de software de ahorro de dosis.

Para la realización del SC se usó una adquisición secuencial de acuerdo con las normas de la SCCT (Society of Cardiovascular Computed Tomography), mientras que, para el protocolo de ATCC se utilizó un protocolo de adquisición retrospectivo.

La información recolectada fue tabulada a través del software Excel y el análisis estadístico se realizó con ayuda del software IBM SPSS Statistics Versión 25.

Se analizaron las variables dosimétricas y la dosis efectiva del score de calcio y angiotomografía por separado.

La variable cualitativa definida en este estudio fue sexo, mientras que, las variables cuantitativas fueron edad, DLP, mAs, kV, DRE; todas estas variables fueron descritas como porcentajes (\%) y desviaciones estándar (DS).

Se empleó la prueba de Test Student para comparar las medias de las variables DLP, $\mathrm{kV}$, mAs en ambos estudios imagenológicos con la dosis media de radiación efectiva.

Finalmente, se calculó la dosis de radiación efectiva utilizando el DLP para cada adquisición de forma independiente, el cual se multiplicó por la constante de Monte Carlo $\left(0,014 m S v \cdot m G{ }^{*} \mathrm{~cm}\right)(6)$ y del DLP total que incluye el topograma, precontrol, control, fases simple y contrastada (Figura 1).

Los valores con $\mathrm{p}<0,05$ se consideraron estadísticamente significativos, empleando un intervalo de confianza de $95 \%$. 


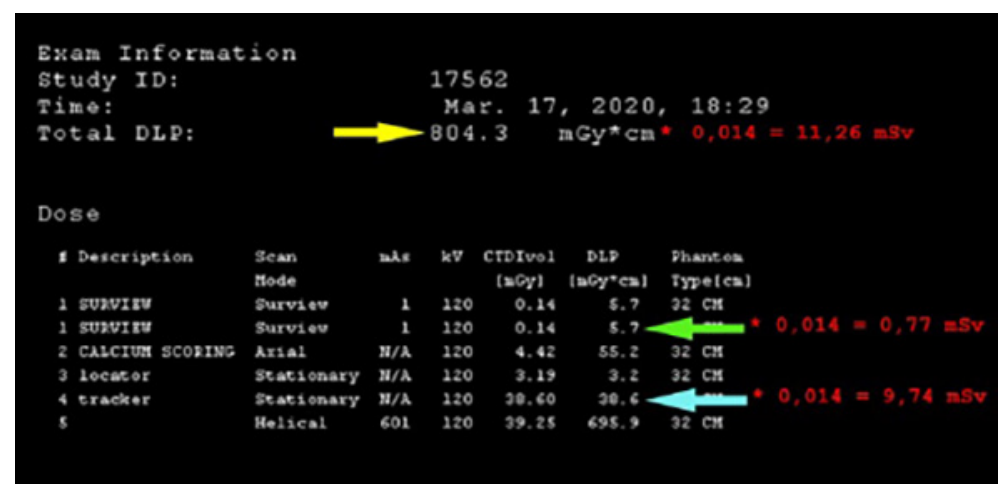

Figura 1: Informe de dosis. La dosis de radiación efectiva se calcula utilizando el DLP total (flecha amarilla), DLP score de calcio (flecha verde), DLP angiotomografía de arterias coronarias (flecha celeste) multiplicados individualmente por el factor de conversión de Monte Carlo $\left(0,014 \mathrm{mGy}{ }^{*} \mathrm{~cm} /\right.$ $m S v)$.

Fuente: propia de los autores.

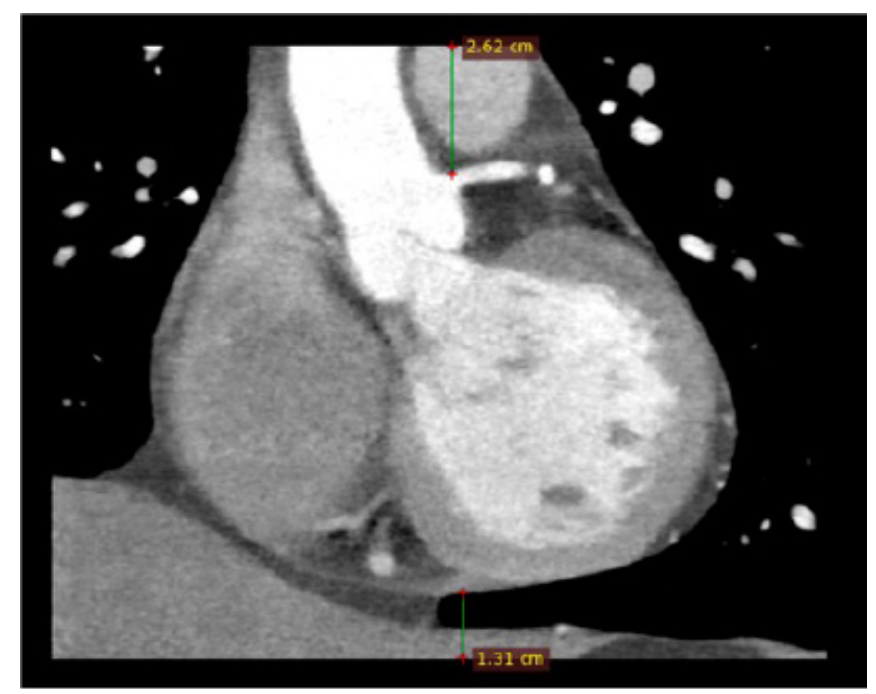

Figura 2: Medida de FOV (medida de campo de visión). (a) campo de visión superior y (b) campo de visión inferior.

Fuente: propia de los autores.

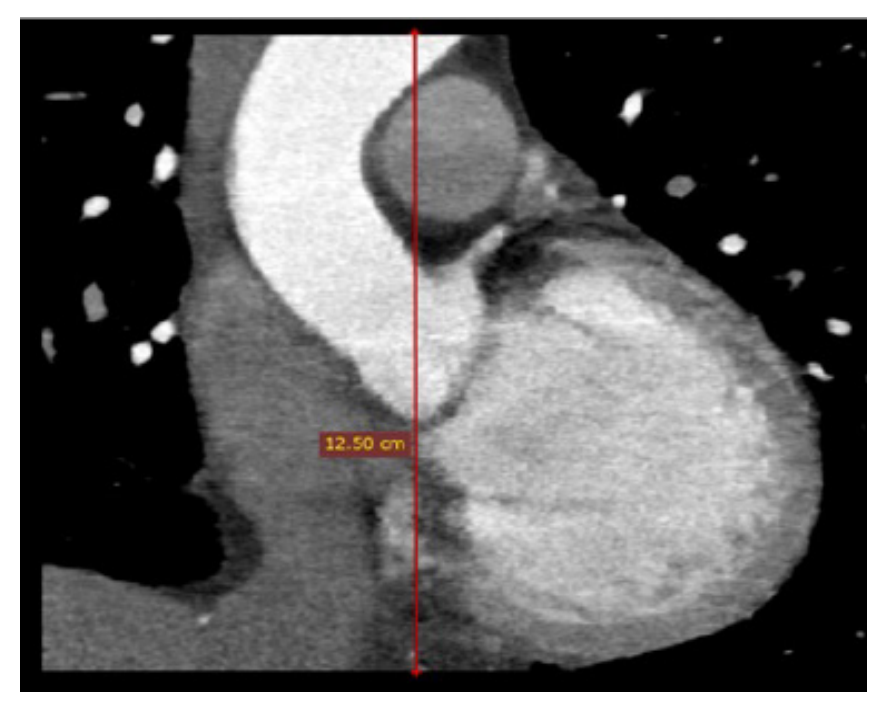

Figura 3: Longitud de adquisición. Medida de la longitud de adquisición de una angiotomografía de arterias coronarias.

Fuente: propia de los autores. 


\section{Resultados}

Se analizaron las tomografías de 106 pacientes [53 mujeres $(50 \%)$ y 53 [hombres (50\%)], con edad pro- medio de 57 años $\pm 16,28$ DS (rango 21-93 años) que cumplieron con los criterios de inclusión. Se obtuvieron un total de 189 estudios, 104 correspondían a SC y 85 a ATCC (Tabla 1).

Tabla 1. Distribución de estudios de acuerdo con el número de pacientes

\begin{tabular}{|c|c|c|c|c|}
\hline N Pacientes & Sexo & Edad media & \multicolumn{2}{|c|}{ Tipo de estudio } \\
\hline \multirow[t]{3}{*}{106} & $H: 53$ & \multirow{2}{*}{57 años } & SC & 104 \\
\hline & $M: 53$ & & ATCC & 85 \\
\hline & & \multicolumn{2}{|c|}{ Número total de estudios } & 189 \\
\hline
\end{tabular}

$\mathrm{N}$ (número).Sexo: H (hombres) y M (mujeres). Tipo de estudio: SC (score de calcio) y ATCC (angiotomografía de coronarias).

Fuente: propia de los autores.

De los 104 pacientes que se sometieron a un score de calcio, 52 fueron mujeres (50\%) y 52 hombres $(50 \%)$, la media de dosis de radiación efectiva (DRE) fue de $0,81 \mathrm{mSv} \pm 0,07 \mathrm{DS}$ y $0,86 \mathrm{mSv} \pm 0,08 \mathrm{DS}$, respectivamente (Tabla 2).
Tanto en hombres como en mujeres la media del kilovoltaje fue de $120 \mathrm{kV}$, la longitud de adquisición media en el sexo masculino fue de $13,86 \mathrm{~cm} \pm 1,39 \mathrm{DS}$ y $12,97 \mathrm{~cm} \pm 1,13$ en el sexo femenino. El DLP (producto longitud dosis) en hombres fue 62,05 $\mathrm{mGy}^{*} \mathrm{~cm} \pm 6,23$ y en mujeres de 57,32 $\mathrm{mGy}^{\star} \mathrm{cm} \pm 5,32$ (Tabla 3).

Tabla 2. Dosis efectiva media según sexo y tipo de estudio

\begin{tabular}{|c|c|c|c|}
\hline Sexo & & SC & ATCC \\
\hline \multirow{3}{*}{ Hombres } & $\mathrm{N}$ & 52 & 41 \\
\hline & DREm & 0,86 & 10,88 \\
\hline & Des.estándar & 0,08 & 1,67 \\
\hline \multirow{3}{*}{ Mujeres } & $\mathrm{N}$ & 52 & 44 \\
\hline & DREm & 0,80 & 10,31 \\
\hline & Des. estándar & 0,07 & 1,43 \\
\hline
\end{tabular}


Tabla 3. Promedio de variables dosimétricas y radiación efectiva según el sexo y tipo de estudio.

\begin{tabular}{|c|c|c|c|c|c|c|c|c|c|c|c|}
\hline \multirow[t]{2}{*}{ Sexo } & & \multicolumn{2}{|c|}{ mAs } & \multicolumn{2}{|c|}{$\mathbf{k V}$} & \multicolumn{2}{|c|}{ LA cm } & \multicolumn{2}{|c|}{ DLP mGy*cm } & \multicolumn{2}{|c|}{ DRE mSv } \\
\hline & & ATCC & S.C & ATCC & S.C & ATCC & S.C & ATCC & S.C & ATCC & S.C \\
\hline & Media & 606,29 & & 120,49 & 120 & 13,75 & 13,86 & 777,73 & 62,05 & 10,88 & 0,86 \\
\hline Hombre & $\mathrm{N}$ & 41 & * & & & & & & & & \\
\hline & Desv. Estandar & 36,78 & & 3,12 & 0 & 1,46 & 1,39 & 119,79 & 6,23 & 1,67 & 0,08 \\
\hline Mujer & $\begin{array}{l}\text { Media } \\
\mathrm{N}\end{array}$ & $\begin{array}{c}603,34 \\
44\end{array}$ & * & 120,91 & 120 & 12,89 & 12,97 & 736,90 & 67,32 & 10,31 & 0,80 \\
\hline & Desv. Estandar & 42,92 & & 4,21 & 0 & 1,68 & 1,13 & 102,34 & 5,32 & 1,43 & 0,07 \\
\hline Total & $\begin{array}{l}\text { Media } \\
\mathrm{N}\end{array}$ & $\begin{array}{c}604,76 \\
85\end{array}$ & * & 120,71 & 120 & 13,31 & 13,41 & 756,60 & 59,68 & 10,59 & 0,83 \\
\hline & Desv. Estandar & 39,87 & & 3,71 & 0 & 1,63 & 1,34 & 112,32 & 6,23 & 1,57 & 0,08 \\
\hline
\end{tabular}

mAs (miliamperaje), kV (kilovoltaje), LA (longitud de adquisición), DLP (producto longitud dosis) y DRE (dosis de radiación efectiva).

*No aplica

Fuente: propia de los autores.

Tabla 4. DLP y DRE total según el tipo/s de estudio que se realizaron los pacientes.

\begin{tabular}{|c|c|c|c|c|c|c|c|}
\hline \multirow[b]{2}{*}{ Sexo } & & \multicolumn{2}{|c|}{ SC } & \multicolumn{2}{|c|}{ ATCC } & \multicolumn{2}{|c|}{ ATCC-SC } \\
\hline & & DLP T & DRE & DLP T & DRE & DLP T & DRE \\
\hline \multirow{3}{*}{ Hombre } & Media & 75,87 & 1,06 & 747,3 & 10,46 & 889,64 & 12,5 \\
\hline & Desv.Estándar & 5,92 & 0,82 & * & & 125,10 & 1,75 \\
\hline & $\mathrm{N}$ & \multicolumn{2}{|c|}{12} & \multicolumn{2}{|c|}{1} & \multicolumn{2}{|c|}{40} \\
\hline \multirow{3}{*}{ Mujer } & Media & 72,24 & 0,94 & 926,70 & 12,97 & 833,13 & 11,66 \\
\hline & Desv. Estándar & 10,84 & 0,15 & * & & 105,51 & 1,47 \\
\hline & $\mathrm{N}$ & \multicolumn{2}{|c|}{9} & \multicolumn{2}{|c|}{1} & \multicolumn{2}{|c|}{43} \\
\hline \multirow{3}{*}{ Total } & Media & 72,24 & 1,01 & 837 & 11,71 & 860,37 & 12,04 \\
\hline & Desv. Estándar & 9,20 & 0,12 & 126,85 & 1,77 & 118,12 & 1,65 \\
\hline & $\mathrm{N}$ & \multicolumn{2}{|c|}{21} & \multicolumn{2}{|c|}{2} & \multicolumn{2}{|c|}{83} \\
\hline
\end{tabular}

SC (score de calcio). ATCC (angiotomografía de coronarias). DLP T (producto longitud dosis total). DRE (dosis de radiación efectiva). N(número).

Fuente: propia de los autores.

Tabla 5. Niveles de dosis efectivas referenciales para score de calcio y angiotomografía de coronarias.

\begin{tabular}{|cc|cccc}
\hline Presente estudio & \multicolumn{2}{|c|}{$\begin{array}{c}\text { Canadian Cardiovascular } \\
\text { Society Position Statement on } \\
\text { Radiation Exposure from } \\
\text { Cardiac Imaging and } \\
\text { Interventional Procedures }\end{array}$} & $\begin{array}{c}\text { Expert Consensus Document on } \\
\text { Optimal Use of lonizing } \\
\text { Radiation in Cardiovascular } \\
\text { Imaging }\end{array}$ \\
\hline SC & ATCC & SC & ATCC & SC & ATCC \\
\hline 0,84 & 10,59 & 3 & 16 & $1-5$ & $8-30$ \\
\hline
\end{tabular}

DRE (dosis de radiación efectiva). SC (score de calcio). ATCC (angiotomografía de coronarias)

Fuente: propia de los autores. 
De los 85 pacientes con estudios de ATCC, 44 fueron mujeres $(51,8 \%)$ y recibieron una DRE media de $10,31 \mathrm{mSv} \pm 1,43$ DS y 41 fueron hombres $(48,2 \%)$ con una DRE media de 10,88mSv $\pm 1,67 \mathrm{DS}$ (Tabla 3).

En los hombres el miliamperio segundo (mAs) medio fue de $606,29 \mathrm{mAs} \pm 36,78 \mathrm{DS}$, la media del kilovoltaje de $120,49 \mathrm{kV} \pm 3,12 \mathrm{DS}$, la longitud de adquisición de $13,75 \mathrm{~cm} \pm 1,46 \mathrm{DS}$ y DLP de $777,73 \mathrm{mGy}^{*} \mathrm{~cm}$ $\pm 119,79$ DS. En las mujeres la media del miliamperio segundo fue $603,34 \mathrm{mAs} \pm 42,92 \mathrm{DS}$, el kilovoltaje fue de $120,91 \mathrm{kV} \pm 4,21 \mathrm{DS}$, la longitud de adquisición de $12,89 \mathrm{~cm} \pm 1,68 \mathrm{DS}$ y DLP de $736,90 \mathrm{mGy}^{*} \mathrm{~cm}$ $\pm 102,34$ DS (Tabla 3).

Tanto el kilovoltaje como el mAs, el DLP y la longitud de adquisición son directamente proporcionales a la dosis de radiación efectiva de los estudios de ATCC.

Por otro lado, el valor de kilovoltaje en todos los estudios de score de calcio fue el mismo $(120 \mathrm{kV})$ tal como sugiere el estudio Multi-Ethnic Study of Atherosclerosis (MESA) $)^{7}$, por lo que no se realizó su correlación con la DRE. En cuanto a las variables DLP y longitud de adquisición se pudo establecer que son directamente proporcionales a la dosis de radiación efectiva (Tabla 4).

El cálculo de la dosis de radiación efectiva total que recibieron los pacientes de acuerdo con el tipo de estudio imagenológico determinó que 21 pacientes se realizaron únicamente el score de calcio de los cuales 12 fueron hombres con un DLP total de $75,87 \mathrm{mGy}^{*} \mathrm{~cm}$ $\pm 5,12$ DS y 9 fueron mujeres con un DLP total de $72,24 \mathrm{mGy}^{*} \mathrm{~cm} \pm 10,84 \mathrm{DS}$.

La DRE total de la población masculina fue de $1,06 \mathrm{mGy}^{*} \mathrm{~cm} \pm 0,82 \mathrm{DS}$ y la correspondiente a la población femenina de $0,94 \mathrm{mGy}^{\star} \mathrm{cm} \pm 0,15 \mathrm{DS}$. Por otro lado, los pacientes que se realizaron únicamente ATCC fueron 2, un hombre con un DPL total de $747,3 \mathrm{mGy}{ }^{*} \mathrm{~cm}$ y una DRE de $10,46 \mathrm{mSv}$, y una mujer cuyo DLP total fue de $926,70 \mathrm{mGy}{ }^{*} \mathrm{~cm}$ recibiendo una DRE total de $12,97 \mathrm{mSv}$.

Un total de 83 pacientes se realizaron ambos estudios de forma simultánea de acuerdo con el protocolo institucional, de ellos 40 fueron hombres con un DLP total de $889,64 \mathrm{mGy}^{*} \mathrm{~cm} \pm 125,10 \mathrm{DS}$, una DRE total de $12,5 \mathrm{mGy}^{*} \mathrm{~cm} \pm 1,75 \mathrm{DS}$ y; 43 mujeres cuyo DLP total fue de $860,37 \mathrm{mGy}^{*} \mathrm{~cm} \pm 118,12 \mathrm{DS}$, con una DRE total de $12,04 \mathrm{mS} \pm 1,65 \mathrm{DS}$ (Tabla 5).

La comparación entre las dosis obtenidas a través del presente trabajo se pudo demostrar que tanto la DRE media de $S C$ de $0,83 \mathrm{mSv} \pm 0,08 \mathrm{DS}$ como la DRE media de ATCC correspondiente a 10,59 $\pm 1,57$ DS son incluso inferiores a las dosis mínimas recomendadas por las organizaciones internacionales. Por otro lado, la DRE total de los pacientes que realizaron únicamente score de calcio fue de $1,01 \mathrm{mSv} \pm 0,12 \mathrm{DS}$, aquellos que se realizaron únicamente ATCC fue de $11,71 \mathrm{mSv}$ $\pm 1,77 \mathrm{DS}$ y aquellos que se realizaron ambos estudios simultáneamente fue de 12,04mSv $\pm 1,65 \mathrm{DS}$ (Tabla 5), demostrándose que la dosis de radiación efectiva se encuentra dentro de los parámetros inocuos establecidos.

\section{Discusión}

La exposición a radiación ionizante constituye un importante problema de salud ya que produce un factor de riesgo de cáncer de por vida, este fenómeno puede atribuirse al espectro electromagnético con altos niveles de energía que tiene la capacidad de eliminar electrones de un átomo y como consecuencia producir daño a las cadenas de ADN. En el informe técnico reciente realizado por el American College of Radiology (ACR) se determinó que el número de estudios de imagen que emiten esta radiación ionizante como la tomografía computarizada (TC) incrementó de 3 a 60 millones en un lapso de 20 años, desde 1985 hasta 2005 en los Estados Unidos ${ }^{8}$.

De forma similar, el Comité de Aspectos Médicos de las Radiaciones en el Medio Ambiente (COMARE), en su décimo sexto informe dio a conocer que en un lapso de 16 años el número de tomografías computarizadas se quintuplicó en el Reino Unido², en nuestro país, aunque no hay información, se cree que estos datos podrían ser similares a los de otros países.

Este notable incremento de estudios tomográficos ha sido considerablemente mayor en los estudios utilizados para valorar enfermedades cardiovasculares debido al creciente número de estas patologías a nivel global. La OMS ha reportado que hasta el año 2019 la primera causa de muerte a nivel mundial fue la cardiopatía isquémica ${ }^{9}$, dato que mantiene concordancia con las estadísticas dadas por el INEC ${ }^{10}$, reportando esta misma patología como la primera causa de muerte en nuestro país.

Los procedimientos de tomografía cardiaca e intervencionista en países como Estados Unidos, son responsables de aproximadamente el $40 \%$ de la dosis de radiación efectiva acumulada anual ${ }^{2}$.

El factor que influye de forma importante para que los estudios cardiacos tengan una mayor dosis de radia- 
ción al compararse con otros estudios tomográficos es la técnica empleada en el protocolo para la adquisición de las imágenes. En la realización de TC cardiaca se debe emplear un equipo con tecnología helicoidal con un tiempo de rotación del tubo de al menos $0.4 \mathrm{~s}$ que permita obtener imágenes de excelente resolución a pesar del movimiento de los latidos cardiacos, facilitando la evaluación de vasos sanguíneos tan finos como las arterias coronarias.

Los pacientes que se sometan a este tipo de estudio requieren cumplir con las siguientes especificaciones: frecuencia cardíaca regular y menor o igual a $60 \mathrm{lpm}$, una función renal normal, capacidad de seguir instrucciones, y tolerancia a los medios de contraste y betabloqueantes, asimismo, se requiere un equipo de al menos 64 detectores.

Se realiza un ECG (electrocardiograma) para sincronizar la adquisición de las imágenes con el ciclo cardíaco. De acuerdo con el objetivo del estudio y los equipos disponibles, existen dos técnicas importantes a considerar: ATCC con gatillado prospectivo y ATCC con gatillado retrospectivo.

La ATCC con técnica de gatillado prospectivo está indicada para el estudio de la anatomía cardiaca y las arterias coronarias, es llevada a cabo en la fase de diástole debido a que en esta hay menor movimiento.

La ventaja de esta técnica es que la calidad de imágenes obtenidas es adecuada mientras la frecuencia cardíaca se mantenga estable y no supere los 60 lpm, además, disminuye la dosis de radiación que recibe el paciente, ya que solo recibe radiación durante la diástole. La desventaja es que no todo paciente tiene frecuencias cardiacas regulares, estables y menores de $60 \mathrm{lpm}$, por lo tanto, no son candidatos para esta técnica.

Para contrarrestar estas limitaciones se ha utilizado la ATCC con técnica de gatillado retrospectivo que, permite evaluar con mejor precisión los movimientos del corazón siendo de utilidad para valorar la función ventrículo valvular y adicionalmente, está indicada en pacientes cuya frecuencia cardíaca es alta e irregular, en nuestra institución se ha establecido el uso del protocolo retrospectivo para todos los pacientes por la gran cantidad de información que brinda al médico.

En esta modalidad se adquiere imágenes durante todo el ciclo cardíaco, si bien permite realizar reconstrucciones en todas las fases y escoger la fase con menor movimiento para evaluar la anatomía cardíaca y las arterias coronarias, también incrementa a la par la dosis de radiación recibida por los pacientes. Para disminuir este efecto colateral, se ha recomendado modular la dosis de radiación total disminuyendo la dosis en fase sistólica hasta un $25 \%$ y manteniendo la dosis completa en fase diastólica ${ }^{11}$.

Esta última técnica ha sido validada por varias instituciones que trabajan con equipos y condiciones similares a las nuestras, por lo tanto, es la que se ha empleado para realizar los estudios imagenológicos de ATCC analizados en este trabajo.

Para el cálculo de la DRE se emplearon factores de conversión según la región anatómica explorada usando el software de simulación de Monte Carlo ${ }^{6}$. Este método utiliza la fórmula $D L^{\star}{ }^{\star} \mathrm{k}$ que se obtiene multiplicando el indicador de dosis DLP por el factor de conversión ( $\mathrm{k}$ ), una constante que tiene un valor de 0,014mSv.mGy*cm.

Si bien, este es el factor de conversión más utilizado actualmente, Trattner et all ${ }^{12}$ en su estudio Factores de Conversión Cardíacos Específicos para estimar la dosis efectiva de radiación a partir del producto dosis-longitud en tomografía computarizada han demostrado que el factor de conversión más apropiado para ATCC es $0,026 \mathrm{mSv} \cdot \mathrm{mGy}^{*} \mathrm{~cm}$, sin embargo, debido a que esta actualización sobre el factor de conversión es reciente y aún no se cuenta con una cantidad considerable de estudios, se usó el factor de conversión de Monte Carlo que hasta la actualidad también ha sido validado obteniendo buenos resulta$\operatorname{dos}^{6}$.

La puntuación de calcio de las arterias coronarias también conocida como score de calcio (SC), es la prueba de imagen por TC sin contraste que ha sido establecida para estratificar el riesgo cardiovascular de pacientes asintomáticos ${ }^{13}$.

La edad media de los pacientes que se realizaron un score de calcio fue de 57,76 años, determinando una dosis radiación efectiva media de $0,83 \mathrm{mSv} \pm 0,08 \mathrm{DS}$ y un kilovoltaje de $120 \mathrm{kv}$.

De acuerdo con las Guías SCCT sobre dosis de radiación y estrategias para la optimización de dosis en TC cardiovascular la dosis de radiación efectiva recomendada debe oscilar entre 1 y $3 \mathrm{mSv}$, el potencial de tubo recomendado es de $120 \mathrm{kV}^{14}$.

Por lo tanto, estas recomendaciones corresponden positivamente con la dosis efectiva encontrada en el presente trabajo. 
La ATCC es una modalidad de estudio imagenológico de gran utilidad para la evaluación de alteraciones de las arterias coronarias en pacientes con antecedente de infarto agudo de miocardio y otras formas síndrome coronario agudo ${ }^{15}$.

A través del presente trabajo se pudo determinar que la dosis de radiación efectiva media fue de $10,59 \mathrm{mSv}$ $\pm 1,57 \mathrm{DS}$, el promedio de kilovoltaje fue de $120,71 \mathrm{kV}$ $\pm 3,71 \mathrm{DS}$, y un miliamperaje de $604,76 \mathrm{mAs} \pm 39,87 \mathrm{DS}$. Allende ${ }^{16}$ en su estudio Calidad de imagen y reducción de dosis en ATCC usando protocolo de baja energía, analizó 20 pacientes en quienes se empleó la técnica retrospectiva determinando que todos los pacientes tuvieron kilovoltaje de $120 \mathrm{kV}$, DLP medio de $599,36 \mathrm{mGy}^{*} \mathrm{~cm} \pm 186,92$ y DRE media de $8,39 \mathrm{mSv}$ $\pm 2,61 \mathrm{DS}$, resultados que se obtuvieron teniendo en cuenta que para la realización de este estudio solo se incluyeron aquellos pacientes que tenían registrado un índice de masa corporal (IMC) de 18,6 a $29,9 \mathrm{~kg} /$ $\mathrm{m}^{2} \mathrm{y} / \mathrm{o}$ enfermedad coronaria en sus historias clínicas lo que disminuyó la DRE media, mientras que, se excluyeron a pacientes obesos.

De forma similar, Capuñay ${ }^{17}$ en su estudio obtuvo una edad media de 60,4 años, un kilovoltaje de $120 \mathrm{kV}$, un miliamperaje de 800 a $1050 \mathrm{mAs}$ y DRE de $10,52 \mathrm{mSv}$ $\pm 1,15 \mathrm{DS}$. Por lo tanto, los resultados obtenidos en los estudios mencionados y los del presente trabajo tienen puntuaciones similares cumpliendo con los límites establecidos, pese a que en nuestra población se incluyeron pacientes con sobrepeso y obesidad mórbida, con la limitante que nuestro estudio no contó con la tabulación de peso y talla que permita aclarar la relación entre la dosis y el IMC 6 .

La DRE recomendada por Canadian Cardiovascular Society Position Statement on Radiation Exposure From Cardiac Imaging and Interventional Procedures ${ }^{18}$ y la dada por Expert Consensus Document on Optimal Use of Ionizing Radiation in Cardiovascular Imaging ${ }^{4}$ son de $16 \mathrm{mSv}$ y de 8 a $30 \mathrm{mSv}$ para ATCC, respectivamente; comparadas con la dosis efectiva media de esta investigación que fue de 10,59mSv se demuestra que se aplicaron correctamente los parámetros dosimétricos para cumplir con el principio de ALARA y adquirir estudios de imágenes de calidad y utilidad diagnóstica.

Del mismo modo, los estudios de score de calcio analizados en los que Canadian Cardiovascular Society Position Statement on Radiation Exposure From Cardiac Imaging and Interventional Procedures ${ }^{18}$ recomendó una DRE de $3 \mathrm{mS} v$ y la sugerida por el Expert Consensus Document on Optimal Use of Ionizing
Radiation in Cardiovascular Imaging de 1 a $5 \mathrm{mSv}^{4}$. Por lo anterior, es importante aclarar que la dosis efectiva que recibieron los pacientes en la totalidad del estudio es la suma de todo el protocolo que incluye el topograma, control, pre-control, fase simple y contrastada, multiplicados por el factor de conversión 0,014 mSv.mGy ${ }^{*} \mathrm{~cm}$, se obtuvo una DRE total de $1,01 \mathrm{mSv}$ para el score de calcio y $11,71 \mathrm{mSv}$ para la ATCC.

Al unir ambas fases la DRE total fue de $12,04 \mathrm{mSv}$, sin embargo, a pesar de presentar valores elevados al compararse con los estudios individuales, se mantiene dentro de los parámetros recomendados, esta particularidad se puede atribuir, a un campo de visión (field of view) estrecho que se limita únicamente a la zona cardiaca, el cual fue de aproximadamente 3,24 $\mathrm{cm}$ por encima de tronco coronario izquierdo y de 2,11 $\mathrm{cm}$ por debajo del pericardio (Figura 2), considerando que el límite recomendado es $2 \mathrm{~cm}$ por encima de la emergencia de las arterias coronarias y $2 \mathrm{~cm}$ por debajo del pericardio ${ }^{19}$; además, el equipo tomográfico que se utilizó tiene un software de ahorro de energía que detecta las frecuencias cardíacas inestables y se desactiva automáticamente otorgando la dosis de radiación completa durante el todo el ciclo cardiaco.

De esta forma, el ahorro de dosis medio fue de 0,88\% \pm 3 ,82DS (rango 0 a $28 \%$ ) confirmando que la preparación previa del paciente que se va a someter a estudios cardíacos es fundamental, ya que aparte de tener un estudio de calidad se puede optimizar la dosis de radiación. Adicionalmente, se pudo comprobar que la longitud de adquisición también es un factor determinante que contribuye a la disminución de la DRE considerando que la media de longitud obtenida en este estudio fue de $13,31 \mathrm{~cm} \pm 1,63 \mathrm{DS}$ para los estudios de ATCC y de $13,41 \mathrm{~cm} \pm 1,34 \mathrm{DS}$ (Figura 3), valores considerablemente inferiores al compararse con los calculados en el estudio The associated factors for radiation dose variation in cardiac $\mathrm{CT}$ angiography cuya longitud de adquisición fue de $15 \mathrm{~cm} \pm 2 \mathrm{DS}^{20}$.

Para la disminución de dosis de radiación en estudios de SC y ATCC se podrían aplicar distintos métodos, ya sea de forma individual o conjunta; la reducción de la corriente del tubo es uno de ellos ya que produce una reducción directa de la dosis de radiación que recibe un paciente, sin embargo, es una decisión que debe meditarse objetivamente teniendo en cuenta que una imagen con mucho ruido, especialmente en pacientes obesos, podría influir en el diagnóstico radiológico, trayendo consigo importantes consecuencias clínicas. 
El radio contraste ruido aumenta al disminuir el voltaje del tubo de rayos $\mathrm{X}$, por lo tanto, para obtener un radio contraste ruido adecuado se debe ajustar la dosis de radiación que recibe el paciente con el kilovoltaje (kV). Disminuir el kV de 120 a $80 \mathrm{kV}$ al realizar una angiotomografía coronaria puede simultáneamente reducir hasta $50 \%$ de la radiación; además, también se recomienda aplicar una dosis controlada mediante electrocardiografía durante una técnica de imagen continua con estudios retrospectivos.

Normalmente, la potencia de emisión se mantiene en su valor nominal durante una fase definida por el operador (en general, fase mesodiastólica a telediastólica), mientras que, durante el resto del ciclo cardíaco, la emisión del tubo se reduce al $20 \%$ de su valor nominal para permitir la reconstrucción de la imagen a lo largo de todo el ciclo cardíaco y de esta forma lograr una reducción en la dosis de radiación del 30-50\%.

La regulación anatómica de la corriente del tubo es una técnica adaptada a la geometría del paciente durante cada rotación del tubo, la emisión del tubo se regula de acuerdo con las características de atenuación tisular en la exploración localizadora o se determina en directo mediante evaluación de la señal de una fila de detectores.

Con esta técnica, es posible reducir la dosis entre 15 y $35 \%$ sin degradar la calidad de imagen, de acuerdo con la región anatómica que se desea explorar. Una variación más sofisticada de la regulación anatómica de la corriente del tubo modifica la emisión del tubo según la geometría del paciente en dirección longitudinal para mantener una dosis adecuada al pasar a otras regiones corporales, por ejemplo, del tórax al abdomen (control automático de la exposición) ${ }^{21}$.

Una de las limitaciones de este estudio fue la falta de información clínica como peso, talla e IMC de cada paciente que permiten establecer una relación mas clara entre la dosis administrada y la contextura del paciente, sin embargo, el factor de conversión de Monte Carlo al ser estandarizado y fácilmente repro- ducible es ampliamente utilizado para calcular la DRE obteniendo resultados aceptables. Además, se debe mencionar que, para tener resultados más precisos, sería necesario incluir una mayor cantidad de pacientes.

Las dosis de radiación efectiva tanto del de SC como de la ATCC, obtenidas a través de todo el protocolo de adquisición de imagen en el presente estudio, no superan las dosis recomendadas por organismos reguladores, sin embargo, una optimización de las variables dosimétricas como el kilovoltaje, mAs y la longitud de adquisición de acuerdo con los factores individuales de cada paciente, puede personalizar un estudio llegando a obtener DRE mucho más bajas pero de buena calidad que permitan una adecuada interpretación radiológica.

\section{Conclusión}

La angiotomografía de arterias coronarias y el score de calcio son dos técnicas relativamente nuevas en tomografía computarizada que nos brindan gran información, sin embargo, estos estudios traen consigo efectos a la exposición a radiación ionizante.

Este factor siempre debe ser considerado en todo estudio radiológico para evitar exponer a los pacientes a dosis excesivas e innecesarias.

La adecuada realización de técnicas de tomografía computada en el corazón con una dosis de radiación dentro de parámetros adecuados es el resultado final de varios pasos que van desde un adecuado control de la frecuencia cardiaca, previo al estudio, así como el ajuste de parámetros de acuerdo con la contextura del pa- ciente ( $\mathrm{kVp}, \mathrm{mAs})$, tanto como la extensión del campo de exploración durante el estudio, los cuales influyen directamente en la dosis de radiación efectiva.

Un adecuado entrenamiento en este tipo de estudios es fundamental en la disminución el riesgo de efectos estocásticos.

Fuente de financiamiento: Recursos propios

Grado de contribución de los autores: Los autores declaran haber contribuido de forma similar en la idea, diseño del estudio, análisis, interpretación de datos y redacción del artículo final.

A.E.P.S: diseño del estudio, recolección, análisis e interpretación de datos, redacción del borrador y del articulo final.

A.S.L.S: idea original, diseño del estudio, redacción del borrador y artículo final

D.S.M.R: análisis e interpretación de los datos, redacción del borrador y artículo final.

P.D.M.S: recolección de datos, redacción del borrador y artículo final.

Conflicto de interés: No existen conflictos de intereses. 


\section{Bibliografía}

1. Brenner D, Hall E. Computed tomography-an increasing source of radiation exposure. The New England journal of medicine [Internet]. 2007 Nov 29 [cited 2021 Aug 1];357(22):2277-84. Available from: https://pubmed.ncbi.nlm.nih.gov/18046031/

2. Williams MC, Stewart C, Weir NW, Newby DE. Using radiation safely in cardiology: what imagers need to know. Heart [Internet]. 2019 May 1 [cited 2021 Aug 1];105(10):798. Available from: /pmc/ articles/PMC6580732/

3. Seguridad del paciente: dosis de radiación en exámenes de rayos X y tomografía computarizada [Internet]. 2019 [cited 2021 Mar 12]. Available from: https://www.radiologyinfo.org/en/info.cfm?p$g=$ safety-xray

4. Hirshfeld JW, Ferrari VA, Bengel FM, Bergersen L, Chambers CE, Einstein AJ, et al. 2018 ACC/HRS/NASCI/SCAI/SCCT Expert Consensus Document on Optimal Use of lonizing Radiation in Cardiovascular Imaging-Best Practices for Safety and Effectiveness, Part 1: Radiation Physics and Radiation Biology: A Report of the American College of Cardiol. Journal of the American College of Cardiology [Internet]. 2018 Jun 19 [cited 2021 Aug 1];71(24):281128. Available from: https://www.sciencedirect.com/science/article/ pii/S0735109718332236?via\%3Dihub

5. Consejo de Seguridad nuclear. Dosis de radiación [Internet]. 2010. Available from: https://www.csn.es/documents/10182/914805/ Dosis de radiación\#: :text=

6. Deak PD, Smal Y, Kalender WA. Multisection CT Protocols: Sexand Age-specific Conversion Factors Used to Determine Effective Dose from Dose-Length Product1. https://doi.org/101148/radiol10100047 [Internet]. 2010 Oct 1 [cited 2021 Aug 1];257(1):15866. Available from: https://pubs.rsna.org/doi/abs/10.1148/radiol. 10100047

7. MESA - Estudio multiétnico de aterosclerosis [Internet]. [cited 2021 Aug 1]. Available from: https://www.mesa-nhlbi.org/

8. American College of Radiology. ACR-SPR-STR Practice parameter for the performance of chest radiography. American College of Radiology [Internet]. 2017;1076(Revised 2017):1-9. Available from: https://www.acr.org/-/media/ACR/Files/Practice-Parameters/chestrad.pdf

9. OMS. Las 10 principales causas de defunción [Internet]. 2020 [cited 2021 Aug 1]. Available from: https://www.who.int/es/newsroom/fact-sheets/detail/the-top-10-causes-of-death

10. INEC. ESTADÍSTICAS DE DEFUNCIONES GENERALES EN ECUADOR. 2019 [cited 2021 Aug 1]; Available from: https://www. ecuadorencifras.gob.ec/documentos/web-inec/Poblacion_y_Demografia/Defunciones_Generales_2019/Presentacion_EDG\%20 2019.pdf

11. Bitar P, Paolinelli P, Furnaro F. Tomografía Computada Cardíaca : Estado Actual Cardiac Computed Tomography : State of the Art Revista Clínica Las Condes [Internet]. 2018;29(1):33-43. Available from: http://dx.doi.org/10.1016/j.rmclc.2017.12.007
12. Trattner S, Halliburton S, Thompson CM, Xu Y, Chelliah A, Jambawalikar SR, et al. Cardiac-specific Conversion Factors to Estimate Radiation Effective Dose from Dose-Length Product in Computed Tomography. JACC Cardiovascular imaging [Internet]. 2018 Jan 1 [cited 2021 Aug 1];11(1):64. Available from: /pmc/articles/ PMC5756125/

13. Chaikriangkrai K, Jhun HY, Shantha GPS, Abdulhak A bin, Sigurdsson G, Nabi F, et al. Coronary artery calcium score as a predictor for incident stroke: Systematic review and meta-analysis. International Journal of Cardiology [Internet]. 2017 Jun 1 [cited 2021 Aug 1];236:473-7. Available from: http://www.internationaljournalofcardiology.com/article/S0167527317300608/fulltext

14. Halliburton SS, Abbara S, Chen MY, Gentry R, Mahesh M, Raff $\mathrm{GL}$, et al. SCCT guidelines on radiation dose and dose-optimization strategies in cardiovascular CT. Journal of Cardiovascular Computed Tomography [Internet]. 2011 Jul 1 [cited 2021 Aug 1];5(4):198. Available from: /pmc/articles/PMC3391026/

15. Nam Ju L, Litt H. Cardiac CT angiography for evaluation of acute chest pain. The international journal of cardiovascular imaging [Internet]. 2016 Jan 1 [cited 2021 Aug 1];32(1):101-12. Available from: https://pubmed.ncbi.nlm.nih.gov/26342713/

16. Allende Núñez F, Araya V K, Madariaga M E, Bitar H P, Paolinelli G P. Calidad de imagen y reducción de dosis en angiotomografía computarizada de arterias coronarias usando protocolo de baja energía. Revista Chilena de Radiologia. 2017;23(3):130-9.

17. Capuñay C, Carrascosa P, Vallejos J, Deviggiano A, Pollono P, García M. Calidad de imagen y dosis de radiación de la angiografía coronaria por tomografía computada multidetector con técnica de adquisición axial con gatillado prospectivo. Revista argentina de radiología [Internet]. 2011;75(3):177-85. Available from: https:// www.redalyc. org/articulo.oa? id $=382538489003$

18. Natarajan MK, Paul N, Mercuri M, Waller EJ, Leipsic J, Traboulsi $\mathrm{M}$, et al. Canadian cardiovascular society position statement on radiation exposure from cardiac imaging and interventional procedures. Canadian Journal of Cardiology [Internet]. 2013;29(11):1361-8. Available from: https://www.onlinecjc.ca/article/S0828-282X(13)00363-2/fulltext

19. Kimura Hayama E, Alexánderson Rosas E, Pale R, Vázquez-Lamadrid J, Talayero Petra JA, Cruz Garciavilla P, et al. Tomografía computada multidetector de arterias coronarias: estado del arte. Parte l: Aspectos técnicos. Arch Cardiol Méx [Internet]. 2007 [cited 2021 Aug 1];77. Available from: http://www.scielo.org.mx/scielo. php?script=sci_arttext\&pid=S1405-99402007000200007

20. Alhailiy AB, Ekpo EU, Kench PL, Ryan EA, Brennan PC, Mcentee $M$. The associated factors for radiation dose variation in cardiac CT angiography. British Journal of Radiology [Internet]. 2019;92(1096). Available from: https://www.ncbi.nlm.nih.gov/pmc/ articles/PMC6886289/

21. Walls Michael C, Rajagopalan Sanjay. Angiografía por tomografía computarizada. In: 14 [Internet]. 2nd ed. 2014 [cited 2021 Aug 1]. Available from: https://www.clinicalkey.es/\#!/content/book/3-s2.0B9788490224380000144?scrollTo=\%23hl0000168. 